\title{
Sensory and memory stimulation as a means to care for individuals with dementia in long-term care facilities
}

This article was published in the following Dove Press journal:

Clinical Interventions in Aging

\section{Michael Mileski \\ Joseph Baar Topinka \\ Matthew Brooks \\ Corie Lonidier \\ Kelly Linker \\ Kelsey Vander Veen}

School of Health Administration, Texas State University, San Marcos, TX, USA

Correspondence: Michael Mileski School of Health Administration, Texas State University, 60I University Drive, San Marcos, TX 78666, USA

Email mileski@txstate.edu

Objective: The primary objective of this study was to identify and further examine the facilitators and barriers of utilizing sensory and memory stimulation as a means to care for individuals with dementia who live in long-term care settings.

Materials and methods: The authors conducted a literature review of 30 academic articles found using the databases such as CINAHL, PubMed, and Academic Search Ultimate from the past 15 years. Facilitator and barrier themes were found within each article and analyzed for their relevance to sensory and memory stimulation therapies and their effects on individuals with dementia.

Results: The most common facilitator was improved communication. The top three barriers were access, staff training, and mixed results.

Discussion: Reminiscence therapy appears to provide a person-centered method of care for those who otherwise have problems communicating. These implementations will be more effective if they have the support of staff and management.

Conclusion: The authors conclude that sensory and memory stimulation therapies have the potential to help improve many dementia-specific issues for individuals living in long-term care settings.

Keywords: long-term care, behavior, management, sensory stimulation, memory stimulation, dementia

\section{Introduction}

Long-term care facilities are often faced with managing multiple residents who exhibit challenging behaviors due to the progressive deterioration of brain cells that causes dementia. ${ }^{1}$ In addition, residents tend to get frustrated while trying to communicate to their caregivers what they need and what problems they are experiencing, which can often lead to agitated behaviors. ${ }^{1}$ The first instinct in these situations often is to seek medication as a means to offset these behaviors. Unfortunately, prescribing too many medications can cause additional issues to arise, such as falls, strokes, or death. ${ }^{2}$ Using non-pharmacological means such as sensory and memory stimulation, however, can promote both physical and emotional comfort to the residents. ${ }^{1}$

Having effective communication between the health care provider and the resident(s) is crucial in making sure that the resident is providing necessary information to the health care provider and that the health care provider is appropriately diagnosing and treating the resident. ${ }^{3}$ Being able to offer alternate avenues for the individuals who are challenged by communicating effectively due to the disease process may help reduce frustration, thus lessening the potential for agitated behavioral disturbances. ${ }^{1}$ 
Furthermore, reminiscence therapy can give the health care provider a better understanding of the resident(s) for whom he or she is caring. Knowledge and understanding of a patient's medical history and cultural background can contribute significantly to optimal patient care and patient satisfaction. ${ }^{3}$ The use of these interventions requires the right staff, with the right knowledge, at the right time, as evidenced from our research on individuals looking at the potential for non-pharmacological interventions.

\section{Background}

For more than 50 years, memory stimulation in the form of reminiscence therapy, introduced by Robert Butler in 1963, has been seen as a need for older adults to help process life events and prepare for death, with added benefits of mental and psychosocial well-being. ${ }^{3,4}$ Paired with the potential benefits of such therapies in the management of behaviors in people with dementia, reminiscence therapy serves dual purposes in this population. These therapies may promote a natural comfort with reminiscence in later life and work to reduce challenging behaviors. ${ }^{4}$ As opposed to the once common thought that storytelling was the result of dementia, Butler shed light on the benefit of using these stories to understand the person with dementia, which can allow us to use effective personalized tools for the individuals in managing behavioral manifestations of dementia. ${ }^{3}$ Non-pharmacological interventions for understanding and managing behavioral manifestations, including reminiscence, life review, sensory stimulation, cognitive stimulation, music therapy, and aromatherapy, among others, have demonstrated positive effects on people living with dementia. ${ }^{5}$

\section{Significance}

The face of long-term care approach is moving away from the biomedical model of care to a more "person-centered approach". This is driven by putting individual residents' wants and needs as the priority for caregiving, so facilities must adapt the way they manage behaviors that are often deemed challenging or as symptoms that need medical management. ${ }^{2}$ The overuse of antipsychotic drugs in the long-term care industry is evidence of the dependence that facilities have on medications to control behaviors in people with dementia, although there is only modest evidence of the efficacy of antipsychotic drugs for this use. ${ }^{2}$ As regulation of these drugs continues to increase, facilities must explore options for the management of behaviors exhibited by people with dementia which encompass the personcentered approach and effectively reduce the behaviors they experience without medications. Non-pharmacological interventions generally provide a reduction in behavioral and psychological symptoms as well as improvement in cognitive function. ${ }^{5}$ To find ways to ensure the quality of care for individuals with dementia residing in long-term care facilities, we must understand the interventions that have the potential to improve services. ${ }^{1}$ These findings can aid facilities as they determine what approaches may be attainable, sustainable, and beneficial to their current and future residents in managing behaviors in a personalized way.

\section{Materials and methods Design}

For this study, the authors completed a systematic review of articles from three databases. PRISMA was used to ensure consistent results from the authors' individualized searches. The initial search was run on June 11, 2017, and the final search was completed on June 17, 2017. Databases utilized by the authors included PubMed, CINAHL, and Academic Search Ultimate (ASU). The initial Boolean string search was expanded with additional relevant terms prior to performing the final search for literature to be reviewed. A final Boolean search string was used across the databases, as shown in Figure 1.

\section{Ethical approval}

This article does not contain any studies with human participants or animals performed by any of the authors. As such, institutional review board (IRB) approval or exemption is not needed, and formal consent is also not required.

\section{Inclusion criteria}

The authors only included articles that were found within the databases such as PubMed, CINAHL, and ASU. Inclusion criteria included English language, human subject only, and academic journals published between June 2002 and June 2017. Across the three databases utilized, duplicate articles were removed from the total number of available articles. Articles were briefly reviewed via examination of abstract to determine whether the information present was germane to the topic being reviewed. The authors have outlined their method of article selection in the PRISMA diagram featured in Figure 1.

\section{Exclusion criteria}

This literature review excludes articles that identified themselves as systematic reviews, meta-analysis articles, or study 


\section{PubMed (MEDLINE)}

\begin{tabular}{|c|}
\hline ("reminiscing" OR "reminisce" OR \\
"recollect" OR "look back on" \\
OR "life review" OR "spiritual \\
life review" OR "recollection" \\
OR "remembrance" OR \\
"nostalgia" OR "sentimental" \\
OR "story" OR "memoir" OR \\
"sensory stimulation" OR \\
"memory stimulation" OR \\
"multisensory" OR "multi-sensory" \\
OR "sensory room" OR "sensory \\
therapy" OR "reminiscence \\
therapy" OR "snoezelen") AND \\
("dementia" OR "alzheimer's" \\
OR "cognitive impairment" OR \\
"memory loss" OR "dementia care" \\
OR "alzheimer's care" OR "delirium") \\
AND ("long-term care" OR "long \\
term care" OR "skilled nursing" \\
OR "skilled nursing facility" OR \\
"nursing home" OR "nursing \\
facility" OR "SNF" OR "dementia \\
care unit" OR "alzheimer's \\
care unit")
\end{tabular}


\section{CINAHL}

("reminiscing" OR "reminice" OR "recollect" OR "look back on" OR "life review" OR "spiritual life review" OR "recollection" OR "rememberance" OR "nostalgia" OR "sentimental" OR "story" OR "memoir" OR "sensory stimulation" OR "memory stimulation" OR "multisensory" OR "multi-sensory" OR "sensory room" OR "sensory therapy" OR "reminiscence therapy" OR "snoezelen") AND ("dementia" OR "alzheimer's" OR "cognitive impairment" OR "memory loss" OR "dementia care" OR "alzheimer's care" OR "delirium" AND ("long-term care" OR "long term care" OR "skilled nursing" OR "skilled nursing facility" OR "nursing home" OR "nursing facility" OR "SNF" OR "dementia care unit" OR "alzheimer's care unit")



ASU



Figure I Criteria for article selection.

Abbreviation: ASU, Academic Search Ultimate.

protocols. Articles that discussed the benefits of sensory and memory stimulation for individuals with brain disorders other than dementia were not included. The authors did not include articles that discuss sensory and memory stimulation as a means to care for individuals with dementia who live in community settings, unless the results were compared directly to the same therapies received by residents of facilities. Bias was not considered by the authors when conducting this study.

\section{Data analyses}

The authors individually reviewed the 30 articles and narrative summaries regarding the use of sensory and memory stimulation as a means to care for people with dementia in long-term care environments, which were extracted from each article. The themes chosen were by consensus of the authors. Those chosen were agreed upon to be ones which provided overarching summary to the facilitators and barriers extracted. The contributing authors selected and agreed upon the themes that were assigned and which theme each facilitator and barrier was assigned to. Information was extracted from the results, and discussion sections of the articles were reviewed. This information was then placed within two matrix tables, one for facilitators and one for barriers, along with their citation occurrence, frequency sum, and frequency percentage. 


\section{Results \\ Study selection}

The article selection process is outlined in the PRISMA diagram in Figure 1. The initial search yielded 364 articles, found across the three databases (PubMed, CINAHL, and ASU), of which 33 appeared to meet all inclusion criteria and were germane to the topic. In addition, three articles were removed after further review indicating they did not meet inclusion criteria. No additional articles were used from the references. A total of 30 articles were used for this review.

\section{Facilitators}

Facilitator themes that support the concept of sensory and memory stimulation as a means to care for individuals with dementia in long-term care facilities consisted of $60 \%$ of total themes presented (66/110). Among the facilitator themes, $71.2 \%$ found that stimulation therapy resulted in "improved communication," "improved behaviors," "improved quality of life," and "improved function" for individuals with dementia living in long-term care facilities. In addition, $18.2 \%$ of facilitator themes found that these stimulation therapies promote person-centered approaches to care, while $10.6 \%$ of facilitator themes illustrate the "ease of implementation" for some forms of sensory and memory stimulation. Table 1 further elaborates on the prominence of facilitator themes within the articles.

Improved communication was found to be the most common facilitating theme, consisting of $15 / 66(22.70 \%)$ of the total facilitator themes found. Improved communication resulted from the enhancement of both verbal and nonverbal communication methods between residents, staff members, and visiting family members. ${ }^{6-16}$ These communication improvements include staff being more accommodating of resident wishes, and feeling more connected to residents, as well as improving their skills for providing care. ${ }^{8,10,16}$ Residents were able to connect and communicate with their peers during leisure times and interact with staff on a more relaxed level as well as engage with others during reminiscence or sensory

Table I Facilitator themes

\begin{tabular}{llll}
\hline Facilitators & Occurrences & Sum & $\%$ \\
\hline Improved communication & $6-16$ & 15 & 22.70 \\
Person-centered approach & $1,9,17-21$ & 12 & 18.20 \\
Improved behaviors & $7,9,11,13,23-28$ & 11 & 16.70 \\
Improved quality of life & $8,17,20-22,26,29,30$ & 11 & 16.70 \\
Improved function & $6,20,31-33$ & 10 & 15.10 \\
Ease of implementation & $12,15,21,23,34,35$ & 7 & 10.60 \\
& & 66 & 100 \\
\hline
\end{tabular}

activities. ${ }^{8,9,11,14}$ Family members were able to feel pride when offering historical life information about their loved ones, and residents were able to express positive feelings as well as reflect and express familiarity about past experiences. ${ }^{7,16}$

As staff implemented the use of sensory and memory stimulation in the lives of their residents, they consistently observed a natural transition toward a more person-centered approach within their respective facilities, a theme consisting of $12 / 66(18.20 \%)$ of the total facilitator themes..$^{1,9,17-21}$ This theme included one-on-one sessions and personalized individual reminiscence, which allowed for staff to have a better understanding and appreciation for the residents for whom they were caring. ${ }^{8,9,19,22}$ As staff was educated and exposed to the personhood of the individuals they were caring for, negative preexisting beliefs about aging and dementia were dispelled, and overall staff attitudes improved. ${ }^{20,21}$

The theme of improved behaviors had a strong presence in positive results as well, totaling 11/66 (16.70\%) of facilitator themes found. Dementia-specific challenging behaviors such as wandering, exit seeking, pacing, verbal agitation, and poor eating habits improved both in the number of episodes and severity of episodes after residents received reminiscence and sensory therapies. ${ }^{7,9,11,13,23-28}$ Childhood images and music had a particularly calming effect on residents who would often describe stories from the images and tap their feet to the music. ${ }^{7}$ While the therapies were actively in use (eg, music played during bath, multisensory stimulation room sessions, and aromatherapy or massage), verbal agitation was reduced, and pacing was greatly improved. ${ }^{7,9,11,13,23-28}$ As a result of improving the residents' overall behavior, the staff members experienced a sense of excitement and were more willing to implement the recommendations. ${ }^{27}$

In addition to improved behaviors, many residents and their visiting family members felt that improved quality of life was evident after receiving these therapies, also consisting of $11 / 66(16.70 \%)$ of the total facilitators identified. Residents felt that expressing themselves through reminiscence allowed staff to understand them on a more personal level. ${ }^{29}$ The staff generally agreed, and many staff members felt a duty to continue the improvement they had seen in the lives of their residents through sensory stimulation. ${ }^{8,17,20-22,26,29,30}$ In addition, residents who participated in the sensory and reminiscence therapy also felt it helped with depression, gave them a purpose in life, allowed them to feel calmer, and prevented loneliness and boredom..$^{17,21,22,26}$ Multisensory exercises can benefit residents with different diseases and disabilities and can increase the communication process between caregivers and residents. ${ }^{26,30}$ 
Closely behind improved behaviors and improved quality of life were the themes of improved function, making up $10 / 66(15.10 \%)$ of the total facilitator themes found. Articles found an increase in cases in which residents' improvements on their own activities of daily living were noted through reminiscence and sensory stimulation, particularly in efforts made to encourage residents with dementia to increase their intake during meal times. ${ }^{31}$ Brief sessions in a multisensory stimulation room left residents feeling stimulated and hungry, and utilizing reminiscence scripts during meal times helped meal times feel person-centered rather than intake centered. ${ }^{6,20,31-33}$ Improvements in autobiographical memory, factual information, and overall cognition were noted with life review process and sensory/motor stimulation. ${ }^{20,32,33}$ The use of life story books also helped people with dementia to recall information from previous therapeutic sessions and build off the previous study to engage in life review. ${ }^{20}$

Many of the sensory and memory stimulation therapies were found to have an ease of implementation, making up $7 / 66(10.6 \%)$ of the total facilitator themes found. Most of these therapies are considered to be cost effective, requiring little resources or complicated skills to implement. ${ }^{34}$ Some were as simple as making a collage from photos that family members were able to provide or building a list of questions to ask residents based on their childhood and young adulthood. ${ }^{12,15,20}$ The easy-to-learn nature of interventions such as aromatherapy or setting out sensory books aided in the ability of intervention to be incorporated into everyday life..$^{12,23,34,35}$ Donations from community members and the use of inexpensive items also removed barriers to implementation. ${ }^{15,21,34}$ While there are some barriers to providing these types of therapies on tight schedules, any attempt to stimulate the resident through reminiscence shows positive correlation toward happier lives. . $^{12,15,21,23,34,35}$ The barriers of implementation are discussed in the "Barriers" section.

\section{Barriers}

The authors identified seven major barriers to implementing sensory and memory stimulation therapies in professional long-term care facilities. Although the total number of barrier themes identified is higher than the number of facilitator themes identified, barriers accounted for only $40 \%$ of total occurrences within the literature reviewed. Authors found a total number of seven barrier themes, while only six facilitator themes were found. Table 2 further elaborates on the challenges that barrier themes present while implementing sensory and memory stimulation therapy in long-term care facilities. "Access," "mixed results," "staff training," and
Table 2 Barrier themes

\begin{tabular}{llll}
\hline Barriers & Occurrences & Sum & $\%$ \\
\hline Access & $\mathrm{II}, 18,2 \mathrm{I}, 23,24,28$ & 9 & 20.50 \\
Staff training & $12,14,19,20,23,24,29$ & 9 & 20.50 \\
Mixed results & $6-8,20,23,30-32$ & 9 & 20.50 \\
Low staff-to-resident ratio & $8,10,1 \mathrm{I}, 13,14,23,25,27$ & 8 & 18.20 \\
Short-term effects & $13,29,32,34$ & 4 & 9.10 \\
Stage of dementia & $16,22,26$ & 3 & 6.80 \\
Negative feelings & 20,22 & 2 & 4.40 \\
& & 44 & 100 \\
\hline
\end{tabular}

"low staff-to-resident ratio" account for $79.7 \%$ of the barrier themes identified within the literature, while "negative feelings," "short-term effects," and "stage of dementia" accounted for the remaining $20.3 \%$ of barrier themes.

Access was cited as a barrier in 9/44 times (20.5\%) and is defined by the authors as a lack of resources to implement the intervention. Significant expenses in implementation for Snoezelen therapy, aromatherapy, and massage are access barriers due to lack of monetary resources needed for continued success. ${ }^{11,23,24}$ For larger therapeutic approaches, such as multisensory stimulation rooms, many facilities found that they simply did not have the time nor items needed to complete their projects. ${ }^{11,21}$ Having the intervention isolated to one room, with a lack of mobility, also became an access issue for residents, and the availability of the interventions could have an effect on their utilization. ${ }^{18,27,35}$ Residents without close friends or family to help relay life history information were not as successful. ${ }^{16}$ Facilities would benefit from relationships with their community so they can rely on donations and volunteers to assist with creating mobile sensory carts with clean, repurposed items. ${ }^{11,18,21,23,24,28}$

In $9 / 44$ barrier themes (20.5\%) of the articles reviewed, residents exhibited mixed results while participating in memory and sensory stimulation. Some residents grew impatient when required to select music or participate for the full 20-30-minute session. ${ }^{7}$ Aromatherapy was not always pleasant for residents, and some did not like picking out their favorite music because the process was too in-depth. ${ }^{7,23}$ One resident was agitated and distracted by reminiscence questions asked by staff during meals, while another resident ate nearly all of her food. ${ }^{31}$ The impact on quality of life was mixed with regard to autobiographical memory approaches with the resident, and it was noted that family or caregivers were required for the highest impacts. ${ }^{6,20}$ The act of reminiscence could not be credited with the improved relationship between caregivers and residents; however, it was believed that the interaction between caregiver and resident was responsible. ${ }^{8}$ Those residents receiving cognitive stimulation 
had better results when they lived in the community versus those who lived in facilities. ${ }^{32}$ The personality of each resident should be taken into consideration when implementing a sensory or memory intervention. . $^{6-8,20,23,30-32}$

It was found in $2 / 44$ barrier themes $(4.4 \%)$ that some residents reacted with negative feelings to reminiscence, memory, and/or sensory therapy when the process took too long to complete or asked too much of them. ${ }^{22}$ The life review process can bring up negative emotions tied to past experiences. ${ }^{20}$ The act of reminiscence was shown to increase depression for some of the people with dementia who took part in the study. ${ }^{22}$ When therapists providing the intervention are properly trained, they can help the resident work through the negative emotions in a healthy way, with little lingering negative effects. ${ }^{20}$

On a similar note, individuals may experience these therapies differently depending on what stage of dementia they are currently experiencing, which was noted in 3/44 barrier themes $(6.8 \%)$. Some residents are not able to verbalize life histories through reminiscing; therefore, staff must rely on nonverbal cues. ${ }^{24}$ Conversation or images may not arouse any sort of reaction from an individual with severe dementia, but touching soft, textured items may help them smile, nod, or feel secure. ${ }^{16,22,26}$ In some instances, researchers were unable to definitively identify the current stage of dementia in a resident to utilize appropriate interventions and accurately measure outcomes. ${ }^{22,26}$

Staff training was cited in 9/44 barrier themes (20.5\%). Staff felt poorly prepared for implementing sensory and memory therapies in facilities as these are not therapies they had received training for while undergoing certification or receiving their degrees. ${ }^{19,24,29}$ Some staff may need to adjust their attitudes to a more resident-centered one, which may be a time consuming process as the staff tries to find the right method/object/approach for each resident. ${ }^{19,29}$ Management must be fully supporting in intervention chosen (sensory interventions, spiritual, reminiscence, etc), as the lack of management support can cause the rest of the team to develop a lack of interest in wanting to use and learn these methods. ${ }^{14,19}$ Facilities may need to adjust schedules and utilize training consultants in order for staff to practice methods. ${ }^{12,14,19,20,23,24,29}$

Overall, a clear barrier found within the articles was a low staff-to-resident ratio, cited in 9/44 barrier themes (18.2\%). The lack of staff available to assist the large number of residents (with varying degrees of dementia) in each facility left staff feeling unable to manage the task. The need for allocated blocks of time to either complete the therapy, or get to know the resident for reminiscence purposes was often difficult. ${ }^{8,13,14,20,23,25,27}$ One-on-one interaction with residents especially in small group settings also requires dedicated personnel. ${ }^{11,14,25}$ Staff members found it difficult to add these types of therapies to their training and schedules when their schedules were already quite demanding with required and regulated work. ${ }^{1,3,8,10,11,13,14,23,25,27}$

Short-term effects were found in 4/44 barrier themes (9.1\%), showing that some therapies only assist in calming the resident during the actual therapeutic session (needing to be performed on a daily basis) or for a short timeframe (6 months or less) after the intervention was completed and did not result in any lingering positive attitudes for an extended period of time..$^{29,32,34}$ In one instance, verbal agitation increased after the intervention, even after basic needs were satisfied. ${ }^{13}$ Temporary improvement was not shown to be proportionate to an enhanced quality of life. . $3,29,32,34^{2}$

\section{Discussion}

Through the information obtained from the literature reviewed, sensory and memory stimulation appear to have a positive effect on both resident outcomes and staff knowledge and caregiving. In the 50 years since reminiscence therapy was introduced, it has become helpful to people with dementia as an effective tool for engaging residents in interactions, memory stimulation, and reduction in agitated behaviors.

Facilitator themes for implementing sensory and memory stimulation include improvements in resident behavior, function (memory, cognitive, and activities of daily living/ instrumental activities of daily living), communication, and quality of life. These themes are all strong indicators of the successes tied to using sensory and memory stimulation to improve the experience of living with dementia in a long-term care facility..$^{6-17,20-25,27,28,30-33}$ In addition to these strong, positive aspects tied to these interventions, ease of implementation from either cost-effective methods or simple training programs that allow for daily incorporation further support the use of these interventions. ${ }^{12,15,21,23,34,35}$ Another positive effect of these interventions was the transition to more person-centered approaches in delivering therapies, care, and developing an appreciation for the person with dementia. , $^{8,9,17-21}$

Barriers to implementing sensory and memory stimulation programs into the care routines of people with dementia often included inadequate staff training and low staff-toresident ratios, with both themes accounting for $37.8 \%$ of identified barriers. ${ }^{8,10-14,20,23-25,27,29}$ These specific themes can be controlled when considering implementation of sensory and memory interventions to help ensure success. 
Access was identified as a barrier on nine occasions, with access issues ranging from interventions being confined to one room, time constraints, cost of implementation, and access to needed resources (ie, family members to obtain life history from). ${ }^{11,18,21,23,24,28}$ Less common barriers included the stage of dementia (4/44) and negative feelings (2/44). 16,20,22,26 $^{-1,2}$ Given the low occurrence of these barrier themes, facilities should still anticipate them in case they arise, but the effects may be minimal, especially in the case of negative feelings, where appropriately trained therapists can help the resident work through his or her negative emotions. ${ }^{16,20,22,26}$ Mixed results arose 9/44 times and often resulted from too much demand on the resident for an extended period of time, activities being too in-depth, or the resident becoming distracted. ${ }^{7,23,31}$ The short-term effects of some interventions were seen as a barrier in 4/44 instances, where the effects were limited to the time of the intervention only, or for less than 6 months after. ${ }^{13,29,32,34}$

Therapies such as reminiscence, life review, and Snoezelen can be better utilized with the identified barriers to implementation being understood by providers. Facilities can use this information to prepare for appropriate staff training, find options for reducing costs through utilizing donated or low-cost materials, and plan for possible time constraints with low staff-to-resident ratios. ${ }^{10-15,19,20,23-25,27,29}$

\section{Limitations}

A limitation to this study is the lack of articles that truly discuss barriers. Indeed, many pieces of research discuss negative aspects; however, there was little research which focused upon the review and exclusion of negative aspects of sensory and/or memory therapies. We can make assumptions from the works which were included in this study, however examining only literature focused on excluding negative aspects of the provision of care would have made this study more advantageous to the reader. The authors also did not look into the research with any specified examination regarding potential mixed effects which might have been in the studies. The authors pulled ideas from the literature reviewed specifically with finding facilitators and barriers in mind, to identify them for use by those in the industry.

\section{Conclusion}

Our results suggest that sensory and memory stimulation can be effective non-pharmacological interventions for providing care and managing behaviors in individuals with dementia who reside in long-term care facilities. These methods of behavioral therapy will be most efficient when staff members receive proper training and support from their management team. If care teams are fully staffed, they will have a better chance of successfully implementing sensory and memory therapies within their facilities. Each facility will face its own respective barriers based on its region, design, and choice of intervention. If staff members in these facilities find a way to overcome or compensate for these barriers, the authors believe that the potential benefits should outweigh the negatives associated with implementation (eg, cost and time dedicated to staff training). Improving the lives of residents may also help facilities maintain a positive environment. It is also apparent that the best approach to implementing these therapy types is by an individualized methodology. There is evidence to show that this personalized approach is necessary to decrease the possibility of negative consequences to individual residents during implementation. As the lives of residents are improved through non-pharmacological reminiscence therapies, their family members and loved ones may feel encouraged to provide positive reviews, which will help a facility maintain full occupancy.

\section{Acknowledgments}

This study received no specific grant from any funding agency in the public, commercial, or not-for-profit sectors.

\section{Author contributions}

All authors contributed toward data analysis, drafting and revising the paper and agree to be accountable for all aspects of the work.

\section{Disclosure}

The authors report no conflicts of interest in this work.

\section{References}

1. Tilly J, Reed P. Evidence on Interventions to Improve Quality of Care for Residents with Dementia in Nursing and Assisted Living Facilities; 2004. Available from: http://www.alz.org/documents/national/ dementiacarelitreview.pdf. Accessed June 9, 2017.

2. Lemay C, Mazor K, Field T, et al. Knowledge of and perceived need for evidence-based education about antipsychotic medications among nursing home leadership and staff. J Am Med Dir Assoc. 2013;14(12):895-900.

3. Tamura-Lis W. Reminiscing - a tool for excellent elder care and improved quality of life. Urol Nurs. 2017;37(3):151-142.

4. Westerhof GJ, Bohlmeijer ET. Celebrating fifty years of research and applications in reminiscence and life review: state of the art and new directions. J Aging Stud. 2014;29:29107-29114.

5. Cammisuli DM, Danti S, Bosinelli F, Cipriani G. Non-pharmacological interventions for people with Alzheimer's disease: a critical review of the scientific literature from the last ten years. Eur Geriatr Med. 2016; 7(1):57-64.

6. Charlesworth G, Burnell K, Crellin N, et al. Peer support and reminiscence therapy for people with dementia and their family careers: a factorial pragmatic randomized trial. J Neurol Neurosurg Psychiatry. 2016;87(11):1218-1228. 
7. Eggert J, Dye C, Vincent E, et al. Effects of viewing a preferred nature image and hearing preferred music on engagement, agitation, and mental status in persons with dementia. SAGE Open Med. 2015;3: 2050312115602579

8. Cooney A, Hunter A, Murphy K, et al. 'Seeing me through my memories': a grounded theory study on using reminiscence with people with dementia living in long-term care. J Clin Nurs. 2014;23(23-24): 3564-3574.

9. Lykkeslet E, Gjengedal E, Skrondal T, Storjord M. Sensory stimulationa way of creating mutual relations in dementia care. Int J Qual Stud Health Well Being. 2014;9(1):23888.

10. Figueiredo D, Barbosa A, Cruz J, Marques A, Sousa L. Empowering staff in dementia long-term care: towards a more supportive approach to interventions. Educ Gerontol. 2013;39(6):413-427.

11. Materne C, Luszcz M, Goodwin-Smith I. Increasing constructive engagement and positive affect for residents with severe and very severe dementia through group-based activities. Aust J Ageing. 2013;33(1): E7-E10.

12. Bate H. Picture perfect: interacting with images. Nurs Res Care. 2012; 14(9):468-474.

13. Bédard A, Landreville P, Voyer P, Verreault R, Vézina J. Reducing verbal agitation in people with dementia: evaluation of an intervention based on the satisfaction of basic needs. Aging Ment Health. 2011; 15(7):855-865.

14. MacKinlay E, Trevitt C. Living in aged care: using spiritual reminiscence to enhance meaning in life for those with dementia. Int J Ment Health Nurs. 2010;19(6):394-401.

15. Stallings J. Collage as a therapeutic modality for reminiscence in patients with dementia. Art Therapy. 2010;27(3):136-140.

16. Luyendyk K. Using life histories to enhance dementia caregiving. Can Nurs Home. 2007;18(1):4-9.

17. Lopes T, Afonso R, Ribeiro Ó. A quasi-experimental study of a reminiscence program focused on autobiographical memory in institutionalized older adults with cognitive impairment. Arch Gerontol Geriatr. 2016;66:183-192.

18. Cohen-Mansfield J, Marx M, Dakheel-Ali M, Thein K. The use and utility of specific nonpharmacological interventions for behavioral symptoms in dementia: an exploratory study. Am J Geriatr Psychiatry. 2015;23(2):160-170.

19. Van Bogaert P, Van Grinsven R, Tolson D, Wouters K, Engelborghs S, Van der Mussele S. Effects of SolCos model-based individual reminiscence on older adults with mild to moderate dementia due to Alzheimer's disease: a pilot study. J Am Med Direc Assoc. 2013;14(7): 528.e9-e13.

20. Subramaniam P, Woods B, Whitaker C. Life review and life story books for people with mild to moderate dementia: a randomized controlled trial. Aging Ment Health. 2013;18(3):363-375.

21. Marques A, Cruz J, Barbosa A, Figueiredo D, Sousa L. Motor and multisensory care-based approach in dementia. Am J Alzheimers Dis Other Demen. 2012;28(1):24-34.
22. O'Shea E, Devane D, Cooney A, et al. The impact of reminiscence on the quality of life of residents with dementia in long-stay care. Int $J$ Geriatr Psychiatry. 2014;29(10):1062-1070.

23. Yang Y, Lee F, Chao H, Hsu F, Wang J. Comparing the effects of cognitive stimulation, reminiscence, and aroma-massage on agitation and depressive mood in people with dementia. J Am Med Dir Assoc. 2016;17(8): 719-724.

24. Bauer M, Rayner J, Tang J, Koch S, While C, O'Keefe F. An evaluation of Snoezelen ${ }^{\circledR}$ compared to 'common best practice' for allaying the symptoms of wandering and restlessness among residents with dementia in aged care facilities. Geriatr Nurs. 2015;36(6):462-466.

25. Maseda A, Sánchez A, Marante M, González-Abraldes I, Buján A, Millán-Calenti J. Effects of multisensory stimulation on a sample of institutionalized elderly people with dementia diagnosis. $\mathrm{Am} \mathrm{J}$ Alzheimers Dis Other Demen. 2014;29(5):463-473.

26. Azcurra D. A reminiscence program intervention to improve the quality of life of long-term care residents with Alzheimer's disease: a randomized controlled trial. Rev Bras Psiquiatr. 2012;34(4):422-433.

27. Lape J. Using a multisensory environment to decrease negative behaviors in clients with dementia. OT Prac. 2009;14(9):9-13.

28. Ward-Smith P, Llanque S, Curran D. The effect of multisensory stimulation on persons residing in an extended care facility. Am J Alzheimer Dis Other Demen. 2009;24(6):450-455.

29. Cruz J, Marques A, Barbosa A, Figueiredo D, Sousa L. Effects of a motor and multisensory-based approach on residents with moderate-to-severe dementia. Am J Alzheimers Dis Other Demen. 2011;26(4):282-289.

30. Heyn P. The effect of a multisensory exercise program on engagement, behavior, and selected physiological indexes in persons with dementia. Am J Alzheimers Dis Other Demen. 2003;18(4):247-251.

31. Cleary S, Hopper T, Van Soest D. Reminiscence therapy, mealtimes and improving intake in residents with dementia. Can Nurs Home. 2013; 23(2):8-13.

32. Picanco Diniz C, Dias e Dias Macedo L, Galdino de Oliveira T, et al. Beneficial effects of multisensory and cognitive stimulation in institutionalized elderly: 12-months follow-up. Clin Interv Aging. 2015;10: 1351-1359.

33. Safavi M, Yahyavi S, Farahani H, Mahmoudi N, Mahboubinia M. The effect of multi-sensory stimulation (MSS) on cognitive status of women with Alzheimer's disease in Fereshtegan elderly care center. J Jahrom Univ Med Sci. 2013;11(2):45-54.

34. Huang H, Chen Y, Chen P, et al. Reminiscence therapy improves cognitive functions and reduces depressive symptoms in elderly people with dementia: a meta-analysis of randomized controlled trials. $J \mathrm{Am}$ Med Direc Assoc. 2015;16(12):1087-1094.

35. Van Weert J, Van Dulmen A, Spreeuwenberg P, Ribbe M, Bensing J. Behavioral and mood effects of Snoezelen integrated into 24-hour dementia care. J Am Geriatr Soc. 2005;53(1):24-33.
Clinical Interventions in Aging

\section{Publish your work in this journal}

Clinical Interventions in Aging is an international, peer-reviewed journal focusing on evidence-based reports on the value or lack thereof of treatments intended to prevent or delay the onset of maladaptive correlates of aging in human beings. This journal is indexed on PubMed Central, MedLine,
CAS, Scopus and the Elsevier Bibliographic databases. The manuscript management system is completely online and includes a very quick and fair peer-review system, which is all easy to use. Visit http://www.dovepress. com/testimonials.php to read real quotes from published authors. 